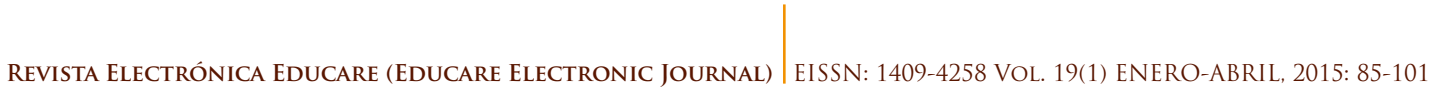

[Cierre de edición 01 de enero del 2015]

doi: http://dx.doi.org/10.15359/ree.19-1.5

URL: http://www.una.ac.cr/educare

CORREO: educare@una.cr

\title{
Desafíos de la formación docente en materia de educación sexual
}

\author{
Challenges of Teacher Training in Sex Education
}

\author{
María Luisa Preinfalk-Fernández ${ }^{1}$ \\ Universidad Nacional \\ Instituto de Estudios de la Mujer \\ Heredia, Costa Rica \\ mlpreinfalk@racsa.co.cr
}

Recibido 30 de octubre de 2014 • Corregido 21 de noviembre de 2014 • Aceptado 22 de noviembre de 2014

\begin{abstract}
Resumen. El presente artículo identifica los vacíos existentes en materia de educación sexual en la población estudiantil del Centro de Investigación en Docencia y Educación de la Universidad Nacional de Costa Rica, y su propósito es contribuir a la toma de decisiones para mejorar la formación de estos estudiantes. Se aplicó un cuestionario estructurado a una muestra por conglomerados desiguales estratificados, compuesta por 242 estudiantes regulares de las carreras de grado. El margen de error fue de $1.5 \%$ y el nivel de confianza del $95 \%$. Los datos se tabularon mediante el software CSPRO y se analizaron con el software R. Asimismo se organizó un grupo focal con la participación de docentes de cursos de sexualidad, con el fin de reflexionar sobre los resultados de la aplicación del cuestionario. Los datos fueron analizados desde el Enfoque de Sexualidad Integral y apuntan a que el estudiantado carece de la información necesaria para ejercer una sexualidad sana, segura y libre de violencia. Esta condición les lleva a situaciones de riesgo ante la baja o nula utilización de anticonceptivos y a la práctica del coito interrumpido; de igual manera, les hace experimentar temores y culpa al mantener relaciones sexuales. Presentan una baja autonomía sexual, lo que les vuelve vulnerables ante situaciones de violencia, con estereotipos y prejuicios, que les llevan a discriminar a otras personas por su orientación sexual o a ser víctimas de ellos. En general, desconocen sus derechos sexuales y reproductivos, y, por tanto, no están en capacidad de exigir su cumplimiento. Estos indicadores constituyen un llamado de atención a las instituciones dedicadas a la formación de docentes para mejorar y fortalecer sus procesos de educación sexual.
\end{abstract}

Palabras claves. Educación sexual, sexualidad, educación superior, universidad.

1 Doctora en Desigualdades e Intervención Social. Directora del Instituto de Estudios de la Mujer. Universidad Nacional de Costa Rica. 
doi: http://dx.doi.org/10.15359/ree.19-1.5

URL: http://www.una.ac.cr/educare

CORREO: educare@una.cr

\begin{abstract}
This article identifies the existing gaps in sex education in the student population of the Center for Research in Teaching and Education of the National University of Costa Rica, and its purpose is to contribute to the decision-making process to improve training for these students. A questionnaire was applied, using a structured sample stratified by unequal clusters, to 242 regular undergraduate students. The margin of error was $1.5 \%$ and the confidence level was 95\%. Data was tabulated using the CSPRO software and analyzed with the R software. A focus group was also held with teachers in sexuality courses in order to reflect on the results of the questionnaire application. Data was analyzed through the Integral Sexuality Approach and suggests that students lack the information necessary to exercise healthy, safe, and violence-free sexuality. This condition leads them to high risk situations because of the rare or lack of contraceptive use and the practice of coitus interruptus and makes them experience fears and guilt when being sexually active. Students have low sexual autonomy, which makes them vulnerable to violent situations and gives them stereotypes and prejudices that lead them to discriminate others for their sexual orientation or to become victims. In general, they are unaware of their reproductive and sexual rights and, therefore, are not capable of demanding them. These indicators should warn teacher training institutions to improve and strengthen their sexual education processes.
\end{abstract}

Keywords. Sex education, sexuality, higher education, university.

En el año 2001, el Estado costarricense declaró la educación sexual obligatoria y transversal en el currículo de la Educación General Básica², con la aprobación, por parte del Ministerio de Educación Pública (MEP) (2004), de las Políticas de Educación Integral de la Sexualidad Humana, reconociendo así la responsabilidad estatal de coadyuvar en esta labor educativa.

Este acto generó una gran expectativa entre la población costarricense, ya que se tenía la esperanza de que, mediante la aplicación del instrumento normativo, se contribuyera a hacer efectivos los derechos de las personas menores de edad en el campo de la educación sexual, dando cumplimiento a las obligaciones establecidas en el Código de la Niñez y la Adolescencia 3 .

\footnotetext{
${ }^{2}$ La educación general básica (EGB) comprende un período de escolarización de 9 años duración, dividido en 3 ciclos: a) EGB I: $1^{\circ}, 2^{\circ}$ y $3^{\circ}$ año (entre los 7 y 9 años), b) EGB II: $4^{\circ}, 5^{\circ}$ y $6^{\circ}$ año (entre los 10 y 12 años), c) EGB III: $7^{\circ}, 8^{\circ}$ y $9^{\circ}$ año (entre los 13 y 15 años) (Preinfalk, 2014).

${ }^{3}$ Artículo 55.- ... Será obligación de los directores, representantes legales o encargados de los centros de enseñanza de educación general básica preescolar, maternal y otra organización, pública o privada, de atención a las personas menores de edad:...c): poner en ejecución los programas de educación sobre salud preventiva, sexual y reproductiva que formule el ministerio del ramo. El incumplimiento de estas obligaciones será sancionado como falta grave para los efectos del régimen disciplinario respectiva". “Artículo 58.-...: En el diseño de las políticas educativas nacionales, el Estado deberá: b): Fomentar los niveles más elevados del conocimiento científico y tecnológico, la expresión artística y cultural y los valores éticos y morales....f): Propiciar la inclusión, en los programas educativos, los temas relacionados con la educación sexual, la reproducción, el embarazo en adolescentes, las drogas, la violencia de género, las enfermedades de transmisión sexual, el SIDA y otras dolencias graves. (Asamblea Legislativa de la República de Costa Rica, 1998, p. 13)
} 
Se implementaron, entonces, nuevos esfuerzos, desde el MEP, por educar en sexualidad a la población joven costarricense; sin embargo, los resultados obtenidos no llenaron los vacíos existentes. Una de las iniciativas más recientes es el Programa de Estudio de Educación para la Afectividad y la Sexualidad Integral (MEP, 2012), que tras superar las resistencias de algunos grupos religiosos, se está desarrollando en las aulas escolares y se confía permita avanzar en esta temática, trascendiendo el "enfoque reduccionista en el abordaje de la sexualidad, de carácter informativo, centrado en aspectos biológicos y preceptos religiosos" que ha caracterizado la educación sexual costarricense (Preinfalk, 2014, p. 2).

Las repercusiones negativas que una educación sexual limitada pueden generar en la población joven han sido ampliamente documentadas, tanto a través de investigaciones, como de estadísticas nacionales. Estas destacan problemáticas que generan preocupación, como el embarazo temprano, fuertemente correlacionado con condiciones de pobreza, no escolarización y desempleo o empleo informal (Collado, 2003), así como con situaciones de rechazo, discriminación y violencia (Carvajal, Preinfalk y Arce, 2008); altos índices de prevalencia de infecciones de trasmisión sexual (ITS) y VIH/SIDA en jóvenes, los cuales están relacionados con una baja utilización del condón, debida a la poca percepción del riesgo en las relaciones sexuales sin protección, desconocimiento, mitos y prejuicios (Fondo de las Naciones Unidas para la Infancia, 2013; Ministerio de Salud, 2011); situaciones de desigualdad, violencia y discriminación hacia grupos en condiciones de vulnerabilidad, asociadas con la prevalencia de estereotipos de género (Escalante, Delvó e Hío, 2011); entre otras.

En este contexto, las instituciones estatales de educación superior enfrentan una doble responsabilidad. Por una parte, educar integralmente a sus estudiantes para que puedan vivir una sexualidad segura, plena y libre de violencia, tal y como lo indican en sus marcos normativos; y por otra, preparar a la población estudiantil de las carreras de educación, para que al insertarse en el mercado laboral cuenten con los conocimientos, habilidades y destrezas necesarias para formar a cientos de niñas, niños y adolescentes en estas temáticas.

El potencial transformador de las universidades se reconoce en las políticas (Política No. 6, Articulación de los Esfuerzos Nacionales), al asignárseles la tarea de coadyuvar en esta labor educativa, a través del desarrollo de acciones formativas dirigidas al futuro profesorado, tales como, cursos especializados, prácticas docentes, investigaciones, trabajos de graduación, proyectos y otros esfuerzos (MEP, 2004).

El objetivo del presente artículo es identificar los vacíos existentes en materia de educación sexual de la población estudiantil de las carreras de educación del Centro de Investigación en Docencia y Educación (CIDE), de la Universidad Nacional de Costa Rica (UNA), con el fin de evidenciar la necesidad de fortalecer las acciones que se desarrollan para su formación. 
doi: http://dx.doi.org/10.15359/ree.19-1.5

URL: http://www.una.ac.cr/educare

CORREO: educare@una.cr

EI CIDE es una instancia de la UNA dedicada a la investigación educativa y a la formación, capacitación y actualización profesional de educadores y educadoras, en los ámbitos de la educación formal y no formal. Imparte diversas carreras a través de sus cuatro unidades académicas, como se detalla a continuación (Preinfalk, 2014, pp. 238-239):

- División de Educología: ofrece formación pedagógica a futuros docentes de educación media (educación secundaria), en las áreas de: Ciencias, Matemática, Español, Estudios Sociales, Inglés, Francés, Educación Musical, Religión, Informática Educativa, Educación Comercial, Filosofía, Educación Física, Ciencias Agrarias y Artes Plásticas, en la modalidad de carreras compartidas.

- División de Educación Básica: forma desde hace más de treinta años docentes en Preescolar, Iy ll ciclo (educación primaria) y Educación Especial. También forma docentes para la Enseñanza del Inglés para I y II ciclo, de manera conjunta con la Escuela de Literatura y Ciencias del Lenguaje.

- División de Educación Rural: ofrece carreras de Diplomado y Bachillerato en Educación Rural con énfasis en I y II ciclo, Licenciatura en Educación I y II ciclo con énfasis en Educación Rural y el posgrado Maestría en Educación Rural Centroamericana.

- División de Educación para el Trabajo: ofrece las carreras de Orientación, Licenciatura en Administración Educativa, Vida Familiar y Social y la Maestría en Gestión Educativa con énfasis en Liderazgo.

\section{La educación sexual integral}

Existen diferentes tipos de abordaje acerca de la educación sexual ${ }^{4}$, desde los cuales se conceptualiza su significado, los fines que persigue, la forma de impartirla, los contenidos a desarrollar, el papel de docentes y estudiantes, entre otros aspectos. Cada uno de estos enfoques se encuentra influenciado por planteamientos sociales, económicos e ideológicos particulares (F. López, 2009).

En el caso de Costa Rica, las posiciones conceptuales sobre la educación sexual que más han permeado el sistema educativo formal son el discurso del silencio, el enfoque positivista biologista y el enfoque moral; de manera reciente se empieza a desarrollar el enfoque de la sexualidad integral (Salas y Campos, 2002). El presente artículo se basa en las premisas de este último.

\footnotetext{
${ }^{4}$ Algunas personas clasifican estos enfoques por sus contenidos: biológicos, morales, sociales, preventivos y personales (Gaudreau, 1985, en F. López, 2009). Otros autores, como Barragán (1991), los clasifican según las teorías o ideologías en que se sustentan: Modelo Represivo-Religioso (Teoría Judeo-Cristiana), Modelo Preventivo (Teoría Burguesa-Tradicional), Modelo Liberal (Teoría Capitalista Permisiva) y Modelo Mercantil (Teoría Productivista) (Preinfalk, 2014, p. 76).
} 
Desde el enfoque de la sexualidad integral se concibe la sexualidad como una manifestación integral de la persona, más allá de su condición biológica. La sexualidad está inserta en el terreno de lo social, de lo individual y de lo colectivo, y se refiere a la forma en que se relacionan las personas, cómo comparten sus pensamientos, vivencias, sentimientos, cómo se dan afecto. Se vincula con la capacidad de sentir, expresar y experimentar (Salas y Campos, 2002; Vargas, 2007).

En el marco de relaciones que establecen las personas, el enfoque de sexualidad integral plantea que una condición necesaria para vivir la sexualidad de manera integral es que esas vinculaciones se desarrollen en un contexto de igualdad y equidad. Esto obliga a incorporar el enfoque de género en los programas de educación sexual: cuestionar el significado social de ser mujer o ser hombre y construir nuevos significados en torno a ello; reconocer los deberes asignados socialmente a cada sexo en la vivencia de la sexualidad; identificar cómo se enseña de manera diferenciada a hombres y mujeres a conceptualizar y vivir su propio cuerpo; puntualizar sobre las prácticas sexuales, creencias, valores y mandatos que norman la sexualidad; valorar las experiencias de cada persona en la reproducción o transgresión de los mandatos existentes y sus posibles consecuencias, etc.

El enfoque de sexualidad integral expone, también, que existen diferentes marcos de referencia para comprender la sexualidad desde lo individual y lo colectivo, al estar mediada por factores biológicos, socioculturales, psicológicos y político-ideológicos que se entrecruzan. Por ello, propone analizar, en el abordaje de la sexualidad, dimensiones como la erótica y el placer; la histórico-cultural; la socialización de género; la ética, en el marco del enfoque de los derechos humanos; la del respeto a la diversidad; y la afectiva.

Otro aspecto que se enfatiza en este enfoque es la responsabilidad que debe asumir el Estado en la promoción de una vivencia integral de la sexualidad. A partir del reconocimiento de que vivir integralmente la sexualidad es un derecho fundamental de toda persona y de la necesidad de recibir una educación sexual adecuada como parte del derecho a la educación, se plantea la obligación del Estado de crear las condiciones para ello.

Sin educación sexual se limita gravemente el ejercicio del derecho a la educación y el goce de los derechos sexuales y reproductivos. Así, el derecho a la educación sexual integral hace parte del derecho de las personas a ser educadas en derechos humanos. (Instituto Interamericano de Derechos Humanos, 2009, citado por Muñoz 2010, p. 30)

La educación sexual se convierte, así, en una herramienta fundamental para el disfrute pleno de estos derechos, proporcionando a las personas -especialmente a niños, niñas, adolescentes y jóvenes-, los conocimientos y habilidades necesarias para la toma de decisiones que les lleven a una vivencia sana, segura y placentera de su sexualidad. 
doi: http://dx.doi.org/10.15359/ree.19-1.5

URL: http://www.una.ac.cr/educare

CORREO: educare@una.cr

\section{Método}

La información que se presenta en este documento se obtuvo mediante la aplicación de técnicas cuantitativas y cualitativas de investigación. En un primer momento se aplicó un cuestionario estructurado a una muestra de 242 estudiantes regulares de las carreras de grado de las diferentes divisiones que conforman el $\mathrm{CIDE}^{5}$. Para ello, se utilizó un diseño muestral de conglomerados desiguales estratificados ${ }^{6}$. El tamaño de la muestra se determinó buscando un error máximo de muestreo de 1.5 puntos porcentuales y una confianza del $95 \%$.

Una vez recopilados y procesados los datos obtenidos mediante la aplicación del cuestionario, se organizó un grupo focal con la participación de docentes que impartieron cursos de sexualidad en la UNA durante el año $2012^{7}$, con el fin de reflexionar sobre esos resultados.

De la población estudiada destaca el hecho de que está compuesta principalmente por mujeres jóvenes, que no han establecido una relación de convivencia permanente con una pareja y profesan la religión católica. Al momento de realizar la encuesta, un $77,5 \%$ del estudiantado reportó haber tenido su primera relación sexual, y de estos, un $87,9 \%$ respondió que en los seis meses anteriores había mantenido relaciones sexuales.

Las carreras donde se concentró el mayor número de personas encuestadas fueron Orientación (22,8\% del total), Educación Rural (17,6\%), Educación Especial (17,1\%), Pedagogía en Educación Preescolar (15,7\%) y Pedagogía en I y II ciclos (6,5\%). Un 51,1\% del estudiantado se encuentra pronto a graduarse e incorporarse como docentes en el sistema educativo formal, ya que un $35,5 \%$ cursa el $5^{\circ}$ nivel de carrera y un $15,5 \%$ el $4^{\circ}$ nivel, considerando que la duración de los bachilleratos es de cuatro niveles y de las licenciaturas es de cinco niveles. Un 48,4\% se encuentra cursando entre el primer y tercer nivel de su carrera.

\section{Resultados}

A continuación se exponen diferentes aspectos relacionados con los conocimientos en sexualidad, vivencias y prácticas sexuales de la población estudiantil del CIDE, que están limitando sus posibilidad de vivir una sexualidad sana, segura y placentera, y que brindan indicios de la necesidad de revisar los esfuerzos en educación sexual que se realizan en el ámbito universitario, orientados a su educación integral y a su formación como docentes.

\footnotetext{
5 El marco muestral fue un listado de la población estudiantil matriculada en el I ciclo lectivo de 2011, en el Campus Omar Dengo de la UNA. En el estudio general se incluyeron las 7 facultades o centros existentes en la UNA, dentro de los cuales se encuentra el CIDE. La muestra a nivel de la UNA fue de 766 estudiantes.

${ }^{6}$ Las probabilidades de selección de los conglomerados se asociaron a grupos de estudiantes (cursos) y la selección de los cursos se definió con base en la proporción de estudiantes matriculados en cada facultad o centro. Las facultades y centros constituyeron los estratos. En el caso del CIDE la muestra se amplificó con el fin de realizar inferencias.
}

${ }^{7}$ Participaron en el grupo focal cuatro docentes, de un total de seis. 


\section{Necesidades de formación}

Se espera que el futuro profesorado, en la labor docente que va a ejercer, trasmita a sus estudiantes conocimientos claros y precisos en materia de sexualidad. Sin embargo, al consultar a esta población sobre diversos temas relacionados con la sexualidad, se denota que un porcentaje importante no dispone de la información necesaria para cumplir satisfactoriamente con esta meta.

Sobre el particular, destacan los siguientes indicadores: a) un 17,4\% del estudiantado manifiesta desconocer cuándo puede quedar embarazada una mujer; b) un 10,2\% indica que no tiene información sobre los métodos anticonceptivos que existen y su forma de uso; c) un $52,8 \%$ desconoce sus derechos sexuales y reproductivos y un $10,6 \%$ afirma conocerlos, pero no logra detallar cuáles son; d) un 21,5\% afirma que no tiene información suficiente sobre las ITS y el VIH/SIDA; e) un 25,4\% desconoce cómo puede contagiarse del Virus del Papiloma Humano.

Lo anterior podría deberse, en gran medida, a la calidad de la educación sexual que el estudiantado recibió en la escuela y el colegio: un 43,6\% califica la educación sexual impartida como mala y muy mala, y un 32,0\% de regular (un 3,5\% no recibió educación sexual). Esto deriva también en que un 54,9\% considere que sus conocimientos en sexualidad están en un nivel medio o bajo.

Sin embargo, aún más preocupante es el hecho de que un 59,4\% expresa que sus conocimientos en sexualidad continúan siendo insuficientes al día de hoy, y un 92,6\% afirma que requiere mayor formación en sexualidad para ejercer la carrera que cursa.

Algunas de sus demandas de formación se dan en torno a temáticas básicas a desarrollar en todo programa de educación sexual: a) métodos y técnicas para abordar en el aula temas de sexualidad con niños, niñas y adolescentes (un $86,5 \%$ lo indica); b) derechos sexuales y reproductivos $(75,1 \%)$; c) salud sexual y reproductiva $(74,0 \%)$; d) respuesta sexual humana (deseo, excitación y orgasmo) (72,2\%); e) métodos contraceptivos y su uso $(69,9 \%)$; f) placer y erotismo (68,9\%); g) anticoncepción de emergencia (64,5\%); h) ITS, VIH/SIDA (63,3\%).

Los indicadores anteriores llevan a reflexionar sobre la necesidad de reforzar la educación en sexualidad que se está brindando a esta población en el ámbito universitario. Al momento de la investigación, por ejemplo, se identificó que en algunas divisiones del CIDE se oferta un único curso especializado en el tema de sexualidad y, en la mitad de los casos, ese curso es optativo, lo cual no asegura la formación del estudiantado.

Asimismo, la mayoría de cursos se imparte desde un enfoque particular ${ }^{8}$, sin profundizar en la diversidad de posiciones existentes; solo en uno de los programas de curso se explicita que se basa en la perspectiva de género, derechos humanos y diversidades, y en otro se indica que

\footnotetext{
${ }^{8}$ Esta afirmación se basa en una revisión de los programas de los cursos identificados.
} 
doi: http://dx.doi.org/10.15359/ree.19-1.5

URL: http://www.una.ac.cr/educare

CORREO: educare@una.cr

busca fortalecer en el futuro profesorado capacidades para construir y desarrollar metodologías adecuadas en el aula para el abordaje de la sexualidad. Este último aspecto constituye la mayor demanda de formación del estudiantado.

Esta dispersión en los enfoques que aborda cada curso (los cursos se ofrecen desde diferentes carreras) no permite al estudiantado conocer y valorar los alcances y limitaciones de cada uno de los planteamientos teóricos existentes, lo cual podría conducir a que en un futuro, cuando el profesorado se encuentre ejerciendo la docencia, reproduzca algunas de estas posturas, que limitan la vivencia de una sexualidad sana y segura. Sobre la educación sexual que reciben, una estudiante de la carrera de Orientación comenta (Preinfalk, 2014, p. 255):

Consideramos que un curso de seis meses en bachillerato no basta, es una sesión a la semana de tres horas diarias y a veces se suspenden las clases... y ¿qué tipo de educación nos están dando? A veces no es tan amplia, no se abarcan todas las orientaciones del deseo, es muy limitada, no se abarcan las infecciones de transmisión sexual... Es importante que a nosotros, la gente del área de educación, se nos dé una buena educación sobre sexualidad (Estudiante de Orientación que participó en grupo focal).

De acuerdo con N. López (2003, p. 13), "la Educación Sexual debe: 1. Informar de forma completa sobre la sexualidad humana para que posibilite una práctica libre, sana y responsable"; sin embargo, esto no es viable si el futuro profesorado no domina los principales conceptos, enfoques y metodologías para desarrollar la temática en el aula.

Los datos anteriores obligan a hacer un alto en el camino y revisar la oferta de formación en materia de educación sexual que se está brindando a esta población. ¿Se está formando al futuro profesorado con los conocimientos, habilidades y destrezas necesarios para facilitar espacios de educación sexual en las aulas escolares, que contribuyan a una formación integral del estudiantado?

\section{Prácticas sexuales en condiciones de riesgo}

Datos de la investigación brindan evidencias de que un importante número de estudiantes del CIDE realiza prácticas sexuales que conllevan un alto riesgo. Dos situaciones relacionadas con este aspecto son: la utilización del coito interrumpido como método seguro de anticoncepción y el infrecuente uso de anticonceptivos en las relaciones sexuales, en especial el condón. Algunas de estas circunstancias podrían haber mediado en el hecho de que un $11,5 \%$ de las estudiantes ha estado embarazada sin desearlo y un $5,9 \%$ de la población estudiantil ha sufrido una ITS. 
Prevalece en el estudiantado la creencia errónea de que el coito interrumpido constituye un método seguro de anticoncepción. Un 49,2\% indica utilizarlo con mayor o menor frecuencia para evitar embarazos, desconociendo que no es una práctica anticonceptiva segura y no le protege de ITS y VIH. Sobre este particular es importante que el futuro profesorado disponga de la información correcta y completa, de manera que pueda negociar sus relaciones sexuales en condiciones seguras y, a la vez, trasmita esos conocimientos a sus estudiantes, colaborando en la erradicación de este tipo de creencias en la población joven.

Por otra parte, la utilización del condón entre la población estudiantil registra los siguientes porcentajes: un 35,5\% no usa condón o casi nunca al mantener relaciones sexuales, un 17,9\% expresa que a veces y un 46,3\% afirma que siempre o casi siempre.

Como se aprecia en los datos anteriores, el porcentaje de uso del condón como forma de protección es bajo entre la población estudiantil del CIDE. Cabe destacar que a nivel general de la UNA la investigación brinda indicios de una tendencia a un menor sobre el uso del condón por parte de las estudiantes mujeres, pese a que ellas registran mayores niveles de conocimiento sobre los métodos anticonceptivos; tendencia que se presenta también en el CIDE.

Sobre el abordaje del tema de la utilización del condón con jóvenes, algunas investigaciones (A. López, 2012; Preinfalk, 2014; Soto, 2006) recomiendan no limitarse a facilitar información, sino profundizar en los factores que están interviniendo en la no utilización de este (entre los que destacan aspectos religiosos y culturales -algunos basados en el género), con el objetivo de comprender cómo les afectan y sus posibles repercusiones. Es conveniente que esta temática se aborde a profundidad en los cursos de sexualidad que se imparten en el CIDE, no solo porque la mayoría de docentes que se están formando son mujeres, sino para desarrollar en el estudiantado las habilidades necesarias para identificar y reflexionar con su alumnado sobre aquellos aspectos que podrían constituir barreras al uso de anticonceptivos.

\section{Ejercicio placentero de la sexualidad}

La sexualidad debe constituir una fuente de bienestar para todas las personas y su disfrute es un derecho inalienable. Sin embargo, en el estudio se identificaron diversos aspectos que limitan el ejercicio pleno y placentero de la sexualidad en la población estudiantil del CIDE. Los resultados que se presentan a continuación se refieren al disfrute en las relaciones sexuales, lo cual no significa que la sexualidad se limite a este ámbito.

Datos del estudio muestran que el miedo y la culpa son dos sentimientos presentes, con mayor o menor frecuencia, en la intimidad del estudiantado: un 34,5\% lo afirma. Asimismo, un $34,2 \%$ indica no sentirse bien cuando mantiene relaciones sexuales. La prevalencia de estos 
doi: http://dx.doi.org/10.15359/ree.19-1.5

URL: http://www.una.ac.cr/educare

CORREO: educare@una.cr

sentimientos podría deberse a diversas razones, entre ellas los preceptos religiosos que maneja esta población. Un 76,8\% de la población profesa la religión católica, y de estos un 71,9\% se considera algo o muy religioso.

En efecto, el hecho de vivir la sexualidad incumpliendo los preceptos dictados por la iglesia católica -virginidad, matrimonio, heterosexualidad, entre otros- podría estar causando tensión en la población joven, generándole miedo, culpa y vergüenza. Sobre este particular, datos de la Primera Encuesta Nacional de Juventud, Costa Rica 2008 (Consejo Nacional de la Política Pública de la Persona Joven, 2008), confirman que, en el caso de la mitad de la población costarricense entre los 15 a 17 años -tanto de zona urbana, como rural-, cumplir con disposiciones religiosas constituye el principal factor por el que no han iniciado relaciones sexuales.

La influencia que puede ejercer la religión en las vivencias de la sexualidad del estudiantado del CIDE es un factor a tomar en cuenta en su formación sexual. Partiendo del respeto a las creencias religiosas de cada estudiante, es necesario abrir espacios para reflexionar sobre su impacto en el disfrute de la sexualidad, la exposición a situaciones de riesgo, limitaciones relacionadas con la construcción de la identidad, entre otras.

\section{Fortalecimiento de la autonomía sexual}

Desde el enfoque de la sexualidad integral, aspectos como los sentimientos y las emociones, la posibilidad de ser feliz, de satisfacer las necesidades espirituales y materiales, de sentirse bien consigo mismas, de relacionarse con las demás personas, de sentir placer y protegerse, entre otros, forman parte del bienestar sexual. A lo anterior, Quirós (2007) agrega una condición básica: "combinar libertad y responsabilidad, en un ambiente de respeto por la dignidad humana" (p. 103). Se debe tener la capacidad de expresarle, a la otra persona, los propios sentimientos y deseos, actuar respetando al otro u otra y recibir, también, su respeto.

La investigación realizada encontró indicios de algunas situaciones que obstaculizan a la población estudiantil del CIDE a vivir libremente su sexualidad: mantener relaciones sexuales por complacer a su pareja, la incapacidad de negarse a mantener relaciones sexuales cuando no se desea o bien mantener encuentros sexuales por presión de sus amistades.

Datos del estudio revelan que un $21,1 \%$ del estudiantado del CIDE ha tenido relaciones sexuales sin desearlo y un $42,6 \%$ por complacer a su pareja. Asimismo, un 2,9\% ha mantenido relaciones sexuales por presión de sus amigos o amigas.

Si se parte del hecho de que un $88,4 \%$ de la población estudiantil del CIDE está compuesta por mujeres, un elemento a considerar que podría estar influyendo en esta situación son los mitos sobre la sexualidad femenina prevalecientes en la sociedad costarricense. Según Basaglia (1985), el mito de la pasividad erótica femenina, basado en que las mujeres son de naturaleza 
pasiva en la sexualidad, obliga a las mujeres a percibirse y ser percibidas como cuerpo-objeto para otros, lo cual les limita a tomar decisiones sobre su propio cuerpo y su sexualidad, permitiendo que sea el otro quien decida por ellas.

Estas nociones, relacionadas con una sexualidad femenina marcada por la pasividad, la restricción y la limitación, forman parte del proceso de construcción de la identidad de las niñas y jóvenes, y como lo explica Lagarde (1994), aprenden que su cuerpo está normado, que no tienen poder de decidir sobre su sexualidad, que son seres dependientes. Los niños, por otra parte, agrega la autora, viven un proceso de construcción identitaria ligado a nociones como el ser propietario del poder y la razón, de los bienes materiales y simbólicos -mujeres, hijos e hijas-, un ser que vive para sí, lo cual legitima su búsqueda de gratificación en todos los ámbitos, incluyendo el sexual.

En este contexto, desarrollar y fortalecer las habilidades de las estudiantes para ejercer autonomía sexual cobra gran relevancia. Esto conlleva:

Desarrollar su capacidad para tomar decisiones sin presión (física, psicológica o social), su capacidad para expresar lo que sienten y desean, su capacidad de actuar respetando los derechos de la otra persona y haciendo respetar los suyos, sus habilidades para negociar con su pareja, amigos(as) y demás personas el cuidado de su salud. Para aprender a negociar su bienestar, el estudiantado debe reconocer que tiene derechos, que sus intereses y deseos son legítimos y deben defenderlos. (Preinfalk, 2014, p. 261)

Como se indica en la cita anterior, otro aspecto que podría estar limitando el ejercicio de la autonomía sexual en la población estudiantil del CIDE es el desconocimiento de sus derechos sexuales y reproductivos, ya que al no saber cuáles son, existe menor posibilidad de que puedan ejercerlos. Datos de la investigación indican que un 52,8\% del estudiantado no logra identificar sus derechos sexuales y reproductivos; a lo cual se suma un 10,6\% de estudiantes que afirma conocerlos, pero no detalla cuáles son. No es casual que al solicitar al estudiantado del CIDE citar sus derechos sexuales y reproductivos hayan omitido mencionar el derecho a vivir libre de violencia sexual.

Considerando las situaciones expuestas que están viviendo las estudiantes y los estudiantes, los espacios que se desarrollen en materia de educación sexual, dirigidos a esta población, deben reconocer que para lograr una vivencia integral de la sexualidad es indispensable cuestionar las premisas en que se ancla el sistema hegemónico: deconstruir el significado de ser mujer y ser hombre en nuestra sociedad; comprender las normas que se le asignan a hombres y a mujeres para vivir su sexualidad (prácticas, creencias y mandatos que norman la sexualidad); identificar las concepciones que se construyen sobre cada cuerpo y sus formas de vivirlo; entre otros aspectos. 
doi: http://dx.doi.org/10.15359/ree.19-1.5

URL: http://www.una.ac.cr/educare

CORREO: educare@una.cr

\section{Prácticas en salud sexual y reproductiva}

El tema de la salud sexual y reproductiva cobra especial relevancia en la educación sexual de las personas jóvenes, no solo por ser un componente integral de su salud general, sino porque este grupo poblacional, por sus prácticas sexuales, es uno de los más vulnerables a sufrir embarazos tempranos y contagiarse del ITS y VIH.

Sobre este aspecto, la investigación profundizó en los conocimientos y prácticas del estudiantado para mantener una adecuada salud sexual y reproductiva. En el caso de las estudiantes, se indagó sobre el autoexamen de mamas y la citología vaginal; mientras que a la población masculina se consultó acerca de la citología masculina y el autoexamen testicular.

Los resultados muestran que las estudiantes registran altos conocimientos en los aspectos señalados; sin embargo, sus prácticas no son frecuentes. Con respecto a la citología vaginal o Papanicolaou, un 91,1\% afirma conocer en qué consiste y sus beneficios, pero únicamente un $52,9 \%$ se la ha practicado. Un $80,1 \%$ indica saber cómo realizarse un autoexamen de mamas y un $46,5 \%$ se lo ha practicado.

En el caso de los hombres, se registran porcentajes muy bajos de conocimientos y prácticas preventivas en salud sexual y reproductiva: 36,8\% conoce la citología masculina, pero 19,4\% se la ha practicado; mientras que un 9,7\% indica conocer qué es el autoexamen testicular y ninguno se lo ha efectuado.

El futuro profesorado tiene un gran potencial transformador en la población escolar, enseñándoles cómo cuidar su salud sexual y reproductiva. Para ello, debe contar con los conocimientos necesarios e incorporar esas prácticas preventivas en su cotidianidad, tareas en las que las universidades pueden contribuir a través de su formación como docentes.

\section{Prácticas discriminatorias}

Otro aspecto investigado que da cuenta de la necesidad de fortalecer la formación en educación sexual de la población estudiantil del CIDE es la poca aceptación de la diversidad sexual, lo cual se refleja en sus concepciones y en la prevalencia de conductas discriminatorias hacia otras personas por su orientación sexual.

Una cuarta parte de la población estudiantil encuestada opina que las prácticas sexuales solo deben darse entre personas heterosexuales; porcentaje que podría ser mayor si se considera que un $27,6 \%$ del estudiantado se mostró ambivalente ante esta afirmación. Mientras que un $8,4 \%$ se manifestó tajante en afirmar que las personas sexualmente diversas no tienen derecho a expresar su orientación sexual; un 17,6\% de la población no se expresó ni en contra ni a favor de esta afirmación. 
Por otra parte, un 10,0\% afirmó que ha discriminado a alguna persona por su orientación sexual y un $4,8 \%$ asintió haber sufrido discriminación por esta causa ${ }^{9}$. Sobre el primer porcentaje es necesario considerar que al estudiantado se le consultó de manera directa si había protagonizado conductas discriminatorias, lo cual podría haber introducido un subregistro de la presencia de este tipo de situaciones, ya que algunas conductas discriminatorias podrían no ser identificadas o percibidas como tales por quien las perpetúa.

El personal docente que participó en el grupo focal coincidió en que este es un aspecto que es necesario reforzar en la formación docente. Sería muy grave que el estudiantado del CIDE, por prejuicios o desconocimiento, permita o contribuya, en un futuro, a reproducir este tipo de conductas en las aulas escolares. De acuerdo con Penna (2012, p. 584), el profesorado debe asumir un rol protagónico para erradicar la homofobia en los centros de estudio "tiene que ser el garante del cambio, el formador del alumnado, el responsable de detectar y actuar contra la violencia homófoba, el encargado de dar una respuesta educativa de calidad ante la diversidad afectivo-sexual".

Para ello, es indispensable abrir espacios en los procesos de formación, con el fin de que el estudiantado reflexione sobre su propia historia sexual, los valores, actitudes y creencias que ha interiorizado (Fallas, Artavia y Gamboa, 2012). Espacios en los que se analice cómo se legitiman las desigualdades y cómo se generan prácticas discriminatorias; en los que se reconozca la diversidad en sus diferentes manifestaciones, no solo sexual, y el invaluable aporte que como personas formadoras pueden brindar en la construcción de sociedades libres de violencia.

\section{Nociones que tienen sobre sexualidad y educación sexual}

Resultados de la investigación señalan que la mayoría de estudiantes del CIDE considera que la educación sexual debe trascender la dimensión biológica o reproductiva (un 17,9\% no se mostró ni a favor ni en contra de este planteamiento) y ligarse a nociones de equidad e igualdad de género $(81,4 \%)$, diversidad (cultural, sexual, religiosa, etc.) $(78,1 \%)$, procesos de socialización $(78,0 \%)$, aspectos culturales $(71,1 \%)$ y de derechos humanos $(68,8 \%)$, entre otros. Para esta población la sexualidad tiene diferentes funciones, como la comunicación, la procreación, el placer, la ternura y el afecto.

\footnotetext{
${ }^{9}$ A nivel de la población estudiantil general de la UNA el estudio registró que son los hombres quienes mayormente realizan actos de discriminación y contra quienes se dirigen este tipo de agresiones, por lo que al estar el estudiantado del CIDE compuesto por un número mayor de mujeres estos porcentajes tienen a ser menores proporcionalmente. A nivel general se registró un $16,5 \%$ de estudiantes perpetradores de situaciones de discriminación y un 7,3\% de estudiantes que las han sufrido.
} 
doi: http://dx.doi.org/10.15359/ree.19-1.5

URL: http://www.una.ac.cr/educare

CORREO: educare@una.cr

Preocupa el hecho de que un $22,5 \%$ del estudiantado considera que la sexualidad es algo instintivo y, por tanto, la educación sexual no es necesaria (un $16,7 \%$ no se muestra ni a favor ni en contra de esta posición). Estas concepciones reflejan posiblemente las pocas posibilidades que el estudiantado ha tenido de reflexionar sobre la importancia de la dimensión sexual en la vida de toda persona y de los aportes que puede brindar un programa de educación sexual.

La investigación identificó, también, que las opiniones del estudiantado se agrupan mayoritariamente en torno a dos posiciones con respecto a lo que consideran debe ser el enfoque de todo programa de educación sexual. La primera posición relacionada con conceptos del enfoque de sexualidad integral, que se denominó en el estudio educación sexual orientada a la formación de personas responsables y autónomas (registrando un promedio general de 90,4) y la segunda posición, asociada a premisas de la religión católica, que se llamó educación sexual basada en la doctrina de la iglesia católica ${ }^{10}$ (con un promedio general de 89,6 ).

La identificación con cada una de las posiciones anteriores varió para cada división del CIDE. Por ejemplo, el estudiantado de Educación Básica se identifica más con una educación sexual para la formación de personas responsables y autónomas (con un promedio de 93,1), mientras que la población estudiantil de Educación para el Trabajo, Educación Rural y Educología, se identifica mejor con una educación sexual cuyo objetivo es trasmitir la doctrina de la iglesia católica (con promedios de 92,5, 87,2 y 86,7 respectivamente).

La prevalencia en el estudiantado de estos enfoques podría explicarse a partir de dos situaciones no excluyentes. Primero, el hecho de que la mayor parte de estudiantes profesa la religión católica y comparte sus premisas y, segundo, que estas nociones fueron las que aprendieron en la educación primaria y secundaria sobre lo que debe ser la educación sexual, aunado al hecho de que quizá no han tenido la oportunidad de conocer a fondos los planteamientos de cada uno de los modelos existentes.

Para el profesorado que ha impartido cursos en sexualidad en la UNA, que participó en el estudio, esta situación responde principalmente a la carencia de cursos de sexualidad en las mallas curriculares o a la inclusión de uno o dos cursos a lo sumo, lo cual limita al estudiantado las posibilidades de manejar visiones más amplias sobre sexualidad, al desconocer los modelos educativos que existen, sus fines, premisas, valores, consecuencias positivas y negativas, etc.

\footnotetext{
${ }^{10}$ Desde esta posición se plantearon al estudiantado "premisas relacionadas con la noción de una educación sexual cuyo objetivo es trasmitir únicamente la doctrina de la Iglesia Católica, restringida a enseñar aspectos biológicos relacionados con reproducción, ya que al hablar a jóvenes sobre aspectos relacionados con la sexualidad se corre el riesgo de incrementar sus relaciones sexuales. A estos planteamientos se agrega una premisa basada en una concepción cultural patriarcal de la sexualidad, que refuerza la doctrina de la Iglesia Católica, que considera que sólo el hombre tiene derecho a vivir de manera activa su sexualidad" (Preinfalk, 2014, p. 227).
} 


\section{Conclusiones}

En Costa Rica, el papel protagónico que asumen las instituciones estatales de educación superior en la formación de profesionales es innegable y en el área de educación esta tarea es trascendental. Así lo indica el Consejo Nacional de Rectores. Oficina de Planificación de la Educación Superior (2011), en el Plan Nacional de Educación Superior Universitaria Estatal 20112015, cuando destaca el especial cuidado que deben tener las universidades en las carreras de educación, "ya que, como formadoras de maestros y maestras, su influencia se adentra profundamente en el futuro de la nación" (p. 78).

La UNA ha asumido esta responsabilidad por más de cuarenta años y hoy se enfrenta a un nuevo reto, fortalecer la formación en sexualidad de su población estudiantil, en especial del futuro personal docente.

Los resultados presentados evidencian que el estudiantado del CIDE está viviendo su sexualidad con carencias de información sobre temas básicos para ejercer una sexualidad sana, segura y libre de violencia; en condiciones de riesgo ante la baja o nula utilización de anticonceptivos (especialmente el condón) y la práctica del coito interrumpido; sintiendo miedo y culpa al mantener relaciones sexuales; con baja autonomía sexual, lo que les vuelve vulnerables ante situaciones de violencia; con estereotipos y prejuicios, que les llevan a discriminar a otras personas por su orientación sexual o sufriendo este tipo de discriminación; desconociendo sus derechos sexuales y reproductivos, y, por tanto, sin capacidad para exigir su cumplimiento; y sin los conocimientos y hábitos necesarios para mantener una buena salud sexual y reproductiva.

Estos resultados reflejan la necesidad de analizar a fondo la oferta académica de las carreras de educación en materia de educación sexual, revisar los contenidos y enfoques de los cursos existentes y asegurarse que cada nuevo o nueva docente que se incorpore en las aulas costarricenses cuente con los conocimientos necesarios y las habilidades y destrezas requeridas para desarrollar temas de sexualidad de una manera integral, libre de mitos y prejuicios, y desde enfoques que promuevan la igualdad, la equidad, la inclusión social y los derechos humanos.

\section{Referencias}

Asamblea Legislativa de la República de Costa Rica. (1998). Código de la Niñez y Adolescencia. Recuperado de http://cpj.go.cr/docs/derechos/codigo-ninez.pdf

Barragán, F. (1991). La educación sexual. Guía teórica y práctica (2ª ed.). Barcelona: Paidós.

Basaglia, F. (1985). Mujer, locura y sociedad (2ª ed.). México: Universidad Autónoma de Puebla. 
ReVISTA ELECTRÓNICA EdUCARE (EDUCARE ELECTRONIC JOURNAL) EISSN: 1409-4258 VOL. 19(1) ENERO-ABRIL, 2015: 85-101

doi: http://dx.doi.org/10.15359/ree.19-1.5

URL: http://www.una.ac.cr/educare

CORREO: educare@una.cr

Carvajal, Z., Preinfalk, M. L.y Arce, P. (2008). La responsabilidad escolar en el embarazo adolescente. Heredia, Costa Rica: Instituto de Estudios de la Mujer.

Collado, A. (julio-diciembre, 2003). Fecundidad adolescente en el gran área metropolitana de Costa Rica. Población y Salud en Mesoamérica, 1(1), 1-39. doi: http://dx.doi.org/10.15517/ psm.v1i1.13914

Consejo Nacional de la Política Pública de la Persona Joven. (2008). Primera Encuesta Nacional de Juventud, Costa Rica 2008: Principales resultados. San José, Costa Rica: Fondo de Población de las Naciones Unidas.

Consejo Nacional de Rectores. Oficina de Planificación de la Educación Superior. (CONARE). (2011). Plan Nacional de la Educación Superior Universitaria Estatal 2011-2015. San José, Costa Rica: Autor. Recuperado de http://163.178.170.71/rid=1L6RSS4N6-4Z2MQD-6GY/ documento\%20planes\%202011-2015.pdf

Escalante, A. C., Delvó P. e Hío, M. (2011). Igualdad y equidad de género en la educación básica pública. San José, Costa Rica: Consejo Nacional de Rectores.

Fallas, M. A., Artavia, C. y Gamboa, A. (2012). Educación sexual: Orientadores y orientadoras desde el modelo biográfico y profesional. Revista Electrónica Educare, 16 (Especial), 53-71. Recuperado de http://www.revistas.una.ac.cr/index.php/EDUCARE/issue/view/418

Fondo de las Naciones Unidas para la Infancia (UNICEF). (2013). Costa Rica. Encuesta de indicadores múltiples por conglomerados 2011: Situación de la niñez y las mujeres. San José, Costa Rica: Ministerio de Salud y UNICEF.

Lagarde, M. (1994). Género e identidades. Metodología de trabajo con mujeres. Quito: FUNDATEC/ UNICEF.

López, A. (setiembre, 2012). Comportamiento responsable de los estudiantes de la Facultad de Estudios Superiores Iztacala en torno al uso de métodos anticonceptivos. Revista Electrónica Psicología Iztacala, 15(3), 843-870. Recuperado de http://www.medigraphic. com/pdfs/epsicologia/epi-2012/epi123d.pdf

López, F. (2009). La educación sexual. Madrid: Biblioteca Nueva.

López, N. (2003). Curso de educación afectivo-sexual. A. Coruña, España: Netbiblo.

Ministerio de Educación Pública (MEP). (2004). Políticas de educación integral de la expresión de la sexualidad humana. San José, Costa Rica: Consejo Superior de Educación.

Ministerio de Educación Pública (MEP). (2012). Programa de Estudio de Educación para la Afectividad y la Sexualidad Integral. San José, Costa Rica: Autor. 
REVISTA ELECTRÓNICA EdUCARE (EDUCARE ELECTRONIC JOURNAL) EISSN: 1409-4258 VOL. 19(1) ENERO-ABRIL, 2015: 85-101

doi: http://dx.doi.org/10.15359/ree.19-1.5

URL: http://www.una.ac.cr/educare

CORREO: educare@una.cr

Ministerio de Salud (2011). Informe de los resultados de la encuesta de salud sexual y reproductiva 2010. San José, Costa Rica: Autor.

Muñoz, V. (2010). Educación sexual. Derecho humano: La piedra y el viento. Montevideo: CLADEM. Recuperado de http://www.codajic.org/sites/www.codajic.org/files/La\%20piedra\%20 y\%20el\%20viento\%20Educación\%20Sexual,\%20Derecho\%20Humano..pdf

Penna, M. (2012). Formación del profesorado en la atención a la diversidad afectivo-sexual (Tesis doctoral). Universidad Complutense de Madrid, España. Recuperada de http://eprints. ucm.es/16718/1/T34011.pdf

Preinfalk, M. L. (2014). La educación sexual en el ámbito universitario: Estudio diagnóstico en la Universidad Nacional, Costa Rica (Tesis doctoral). Universidad Pablo de Olavide, España.

Quirós, E. (2007). Módulo para la promoción de una sexualidad segura, solidaria y desde el cuidado mutuo en la adolescencia. Heredia, Costa Rica: Instituto de Estudios de la Mujer.

Salas, J. M. y Campos, Á. (2002). El placer de la vida. Sexualidad infantil y adolescente: Su pedagogía a cargo de personas adultas. San José, Costa Rica: Lara Segura Editores.

Soto, V. (2006). Factores asociados al no uso del condón: Estudio en adolescentes y adultos jóvenes de Chiclayo. Anales de la Facultad de Medicina, 67(2), 152-159. Recuperado de http://www.scielo.org.pe/pdf/afm/v67n2/a08v67n2.pdf

Vargas, R. (2007). La salud sexual y la salud reproductiva de la población estudiantil de la Universidad Nacional. Heredia, Costa Rica: Instituto de Estudios en Población.

\section{Cómo citar este artículo en APA:}

Preinfalk-Fernández, M. L. (enero-abril, 2015). Desafíos de la formación docente en materia de educación sexual. Revista Electrónica Educare, 19(1), 85-101. doi: http://dx.doi.org/10.15359/ree.19-1.5

Nota: Para citar este artículo en otros sistemas puede consultar el hipervínculo "Como citar el artículo" en la barra derecha de nuestro sitio web:

http://www.revistas.una.ac.cr/index.php/EDUCARE/index 